\title{
Splitting function measurements for Earth's longest period normal modes using recent large earthquakes
}

\author{
Arwen Deuss, ${ }^{1}$ Jeroen Ritsema, ${ }^{2}$ and Hendrik van Heijst ${ }^{3}$ \\ Received 9 November 2010; revised 22 December 2010; accepted 3 January 2011; published 17 February 2011.
}

[1] Recent megathrust earthquakes, such as the 23 June 2001 Peru event, the Sumatra events of 2004 and 2005 and the 27 February 2010 Chile event, have given us the opportunity to measure splitting of the longest period normal modes. We use wave spectra to make robust measurements for modes ${ }_{0} S_{2},{ }_{0} S_{3},{ }_{0} S_{4},{ }_{2} S_{1}$ and ${ }_{1} S_{2}$. Singlet frequencies of these modes have been measured previously using gravimeters, but here we use seismic records to observe splitting functions for ${ }_{0} S_{2}$ and ${ }_{2} S_{1}$ for the first time. Cross-coupling with nearby modes is included to account for ellipticity and rotation of the Earth and results in significantly improved splitting function measurements for ${ }_{0} S_{3},{ }_{0} S_{4}$ and ${ }_{1} S_{2}$ compared with previous studies. The new splitting function measurements can easily be implemented in future tomographic modelling of aspherical velocity and, particularly, density structure. Citation: Deuss, A., J. Ritsema, and H. van Heijst (2011), Splitting function measurements for Earth's longest period normal modes using recent large earthquakes, Geophys. Res. Lett., 38, L04303, doi:10.1029/2010GL046115.

\section{Introduction}

[2] The splitting of Earth's longest period free oscillation spectra places important constraints on the structure of Earth's mantle and core. It is well recognised that splitting functions have the potential to provide density variations in the Earth's mantle [Ishii and Tromp, 1999; Trampert et al., 2004], but progress has been limited by the available data [Romanowicz, 2001]. Improved splitting function measurements may provide the answer, in particular for the longest period normal modes which are most strongly sensitive to density structure. For some of these modes (i.e., ${ }_{2} S_{1}$ and ${ }_{0} S_{2}$ ), splitting functions have not been available and others (i.e., ${ }_{1} S_{2},{ }_{0} S_{3}$ and ${ }_{0} S_{4}$ ) were measured with large uncertainty using a small number of spectra [He and Tromp, 1996; Resovsky and Ritzwoller, 1998].

[3] The Sumatra event of 26 December $2004\left(M_{w}=9.0\right)$ made it possible to observe singlet frequencies for ${ }_{2} S_{1}$ and ${ }_{0} S_{2}$ using gravimeters [Rosat et al., 2005] and seismometers [Roult et al., 2010]. The 27 February 2010 Chile event $\left(M_{w}=8.8\right)$ was almost as large and provided us with another opportunity to measure splitting functions for ${ }_{2} S_{1}$ and ${ }_{0} S_{2}$ for the first time using only seismic records. In comparison

\footnotetext{
${ }^{1}$ Bullard Labs, University of Cambridge, Cambridge, UK.

${ }^{2}$ Department of Geological Sciences, University of Michigan, Ann Arbor, Michigan, USA.

${ }^{3}$ Department of Earth Sciences, University of Oxford, Oxford, UK.
}

Copyright 2011 by the American Geophysical Union. 0094-8276/11/2010GL046115 with previous studies, the new events will more than double the available spectra for modes ${ }_{1} S_{2}$ and ${ }_{0} S_{3}$.

\section{Theory and Method}

[4] Normal modes are standing waves along the surface and radius of the Earth and thus only exist for discrete frequencies. Spheroidal modes ${ }_{n} S_{l}$ involve P-SV wave motion and are characterized by their radial order $n$ and angular order $l$. Each spheroidal mode multiplet ${ }_{n} S_{l}$ consists of $2 l+1$ singlets with azimuthal order $m$ in the range $-l, . ., l$. These singlets have the same frequency (i.e., are degenerate) for a spherically symmetric, isotropic, non-rotating Earth model such as the Preliminary Reference Earth Model (PREM) [Dziewonski and Anderson, 1981]. Rotation, ellipticity, anisotropy and heterogeneous structure remove the degeneracy, thereby splitting the singlets into different frequencies.

[5] The splitting of modes can be completely described using the splitting function approach [Woodhouse and Giardini, 1985; He and Tromp, 1996; Resovsky and Ritzwoller, 1998]. Splitting function coefficients are denoted $c_{s t}$ and depend on the anisotropic and heterogeneous structure in the Earth,

$$
c_{s t}=\int_{0}^{a} \delta m_{s t}(r) K_{s}(r) d r+\sum_{d} \delta h_{s t} H_{s}^{d}
$$

where $s$ is the angular order and $t$ the azimuthal order of the spherical harmonic used to describe the structure in the Earth. $\delta m_{s t}$ and $\delta h_{s t}$ are the coefficients of Earth's heterogeneity (compressional velocity $V_{p}$, shear wave velocity $V_{s}$ and density $\rho$ ) and discontinuity $(d)$ topography in terms of spherical harmonics and $K_{s}(r), H_{s}^{d}$ are known kernels [Woodhouse, 1980]. The splitting function coefficients can be visualized by a splitting function map, i.e.,

$$
F(\theta, \phi)=\sum_{s, t} c_{s t} Y_{s}^{t}(\theta, \phi)
$$

where $Y_{s}^{t}(\theta, \phi)$ are the complex spherical harmonics following the normalization of Edmonds [1960].

[6] Splitting functions are measured from the spectra of large earthquakes. We measure splitting functions by nonlinear iterative least squares inversion [Tarantola and Valette, 1982] and use the same method as Li et al. [1991]. We start our inversions from PREM [Dziewonski and Anderson, 1981], taking into account only the cross-coupling with nearby modes due to ellipticity and rotation. Thus we prevent the choice of starting model from influencing the results for aspherical structure. We invert for the normal mode centre frequency (i.e., $c_{00}$ ) and quality factor jointly with the splitting function coefficients. For each inversion, we use a range of damping parameters, encompassing several orders of 
Table 1. Normal Mode Centre Frequency and Quality Factor Measured in This Study Compared With PREM Values and the Total Number of Records and Events Used. Misfit for PREM (Including Ellipticity and Rotation) and for the Splitting Functions are Also Shown

\begin{tabular}{|c|c|c|c|c|c|c|c|}
\hline & \multicolumn{3}{|c|}{ PREM } & \multicolumn{3}{|c|}{$c_{s t}$} & \multirow{2}{*}{$\begin{array}{l}\text { Num. } \\
\text { rec(ev) }\end{array}$} \\
\hline & $\mathrm{f}(\mu \mathrm{Hz})$ & Q & misf. & $\mathrm{f}(\mu \mathrm{Hz})$ & Q & misf. & \\
\hline${ }_{0} S_{2}$ & 309.28 & 510 & 0.26 & $309.48 \pm 0.05$ & $580 \pm 75$ & 0.22 & $78(08)$ \\
\hline${ }_{0} S_{3}$ & 468.56 & 417 & 0.21 & $468.44 \pm 0.03$ & $404 \pm 15$ & 0.18 & $364(37)$ \\
\hline${ }_{0} S_{4}$ & 647.08 & 373 & 0.26 & $646.76 \pm 0.03$ & $373 \pm 10$ & 0.15 & $578(56)$ \\
\hline${ }_{1} S_{2}$ & 679.86 & 310 & 0.23 & $679.91 \pm 0.05$ & $327 \pm 5$ & 0.18 & $136(09)$ \\
\hline${ }_{2} S_{1}$ & 403.95 & 397 & 0.60 & $404.17 \pm 0.04$ & $414 \pm 70$ & 0.48 & $32(02)$ \\
\hline
\end{tabular}

magnitude. We use norm damping; the damping parameter is determined using cross validation and trade-off curves that display misfit as a function of damping. We only present measurements for which lowering the damping in our inversion does not anomalously increase splitting function coefficients. We model each splitting function up to its maximum sensitive angular order $s_{\max }=2 \cdot l$. However, we find that for most modes reported here only the coefficients up to $s=2$ were robust. We use cross-validation to determine standard error boundaries, leaving out different events in different runs.

\section{Data}

[7] We found that events with $M_{w} \geq 8.0$ produce spectra with longest period normal modes well above noise level. Mode ${ }_{2} S_{1}$ was the most difficult to observe, but we observed clear spectral peaks for the 2004 Sumatra and the 2010 Chile events. Neither of these events has been used before to measure splitting functions. For the other modes, we were able to use a larger number of events, and in particular the 23 June 2001 Peru event $\left(M_{w}=8.4\right)$ and the 2005 Sumatra event $\left(M_{w}=8.6\right)$ provided high-quality spectra. Modes ${ }_{0} S_{3}$ and ${ }_{0} S_{4}$ were observed in over 300 spectra from over 30 events (Table 1). The long source duration of the 2004 Sumatra event has been accounted for by multiplying the frequency spectrum with the best-estimate frequency spectrum of the normal mode source time function [Park et al., 2005].

[8] Figure 1 shows the spectrum at BFO (Black Forest Observatory, Germany) from the 27 February 2010 Chile event for the modes measured in this study. Mode ${ }_{2} S_{1}$ can be seen above the noise level around $0.41 \mathrm{mHz}$. The other modes measured in this study $\left({ }_{0} S_{2},{ }_{0} S_{3},{ }_{0} S_{4}\right.$ and $\left.{ }_{1} S_{2}\right)$ have larger amplitudes. The longest period split modes have up to now been treated as isolated in the measurement of splitting functions. However, presence of ${ }_{0} T_{2}$ and ${ }_{0} T_{3}$ peaks on the vertical component spectra demonstrates that even for the longest period normal modes, cross-coupling to nearby modes due to rotation and ellipticity of the Earth should not be ignored [Dahlen and Sailor, 1979; Zürn et al., 2000; Deuss and Woodhouse, 2001].

[9] For each of the modes we tested the quality of our measurements by comparing splitting functions derived with and without the 2004 Sumatra spectra. Only for mode ${ }_{0} S_{2}$, the longest period mode in our data set, we excluded the Sumatra event as this event destabilized the inversion.
For all other modes, the Sumatra event was included in the inversion, but was left out in one of the cross-validation runs to determine the error boundaries.

\section{Splitting Function Observations}

[10] For the five longest period spheroidal normal modes, the centre frequency, quality factor $Q$ and corresponding PREM model values are shown in Table 1. Also indicated are the misfit for PREM and the splitting functions. Note that the misfit for all modes is significantly lower after adding splitting function measurements. Even though the overall misfit for ${ }_{2} S_{1}$ is large due to its low signal to noise ratio, the data quality is sufficiently high to measure its splitting function (Figure S1 of the auxiliary material). ${ }^{1}$

\subsection{Splitting Function Maps}

[11] Figure 2 shows the splitting function maps (i.e., equation (2)) for the modes, compared with predictions for mantle model S20RTS [Ritsema et al., 1999] and crustal model CRUST5.1 [Mooney et al., 1998] (hereafter called 'model predictions'). All modes are shown up to angular order $s=2\left({ }_{0} S_{4}\right.$ to $\left.s=4\right)$, even though they have all been measured up to their $s_{\max }$ value. While some previous studies [e.g., He and Tromp, 1996] have published splitting function coefficients up to $s=4$ for ${ }_{0} S_{3}$ and ${ }_{1} S_{2}$, we found that it was difficult to constrain these coefficients without heavily damping towards a starting model such as S20RTS.

[12] Our splitting function measurement for mode ${ }_{0} S_{2}$ (Figure 2a) has positive frequency anomalies in the midPacific and under Africa in agreement with model predictions (Figure 2b). The other two fundamental modes ${ }_{0} S_{3}$ and ${ }_{0} S_{4}$ show the characteristic 'ring around the Pacific' pattern, where the splitting functions have positive frequency anomalies (blue regions in Figures $2 \mathrm{c}$ and $2 \mathrm{e}$ ). This pattern is well known from splitting function observations for modes primarily sensitive to mantle structure. It is interpreted as being due to subduction zone regions which have larger than average velocity anomalies and agrees well with the model

${ }^{1}$ Auxiliary materials are available in the HTML. doi:10.1029/ 2010GL046115.

Station BFO, 27 February 2010, $M_{w}=8.8$ Chile event

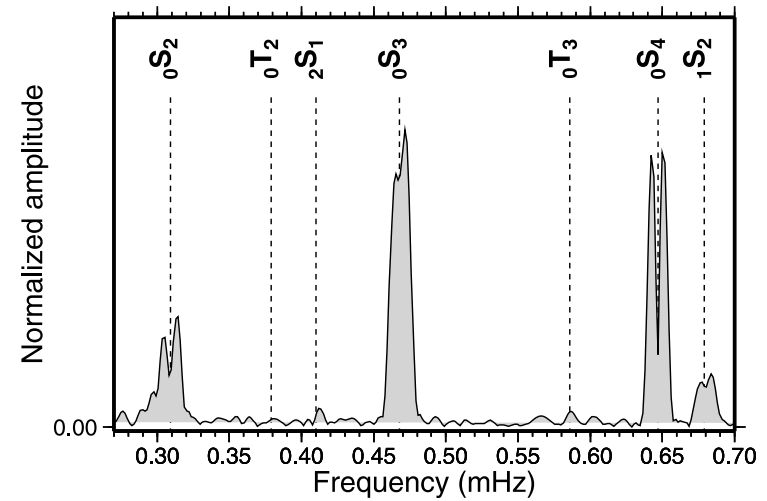

Figure 1. Observed spectrum for the Chile event of 27 February 2010 for the vertical component recording of 80 hours length at seismic station BFO. The high signal to noise ratio makes mode ${ }_{2} S_{1}$ visible in a single seismic record. 

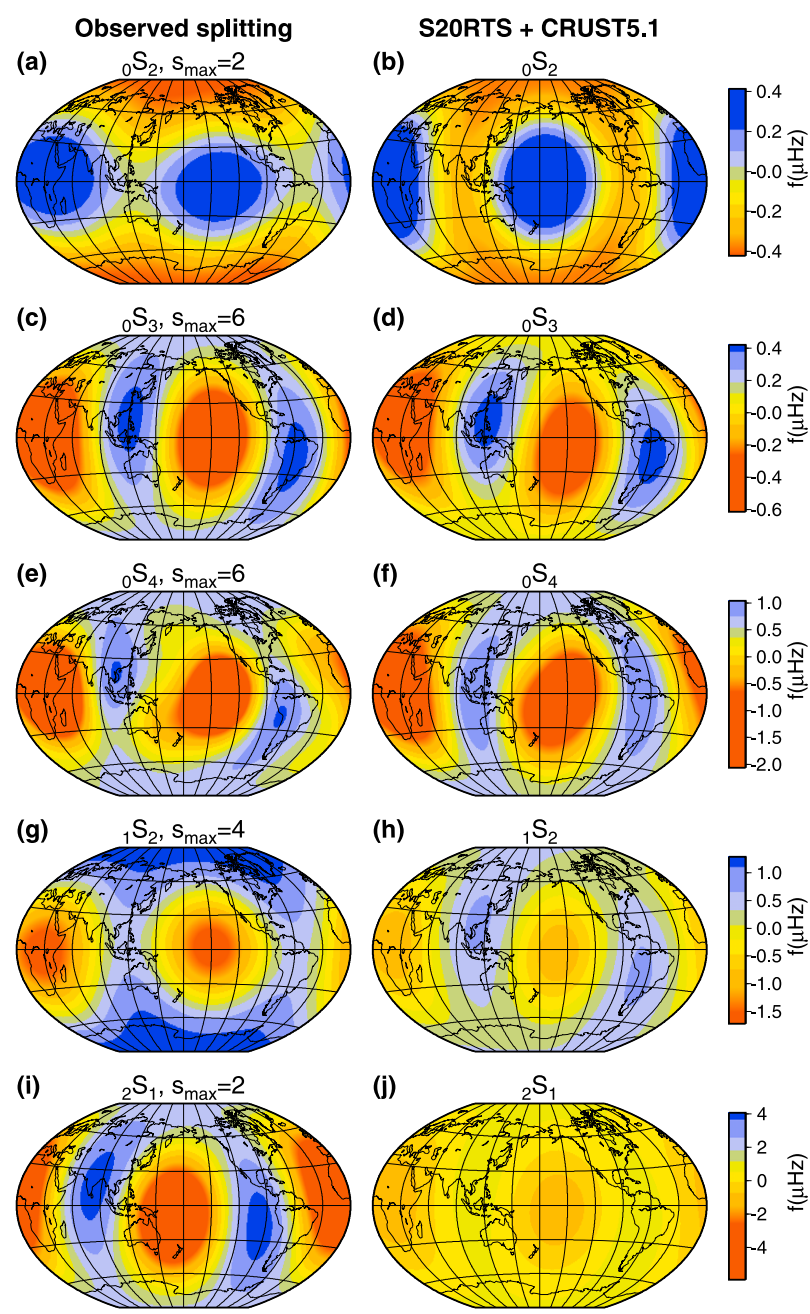

Figure 2. $(\mathrm{a}-\mathrm{j})$ Observed splitting functions and the corresponding predictions for mantle model S20RTS and crustal model CRUST5.1. The modes have been measured up to angular orders $s_{\max }$, but we plot the splitting functions up to $s=2\left({ }_{0} S_{4}\right.$ to $\left.s=4\right)$.

predictions (Figures 2d and 2f). Mode ${ }_{1} S_{2}$ (Figure 2g) shows a 'zonal pattern' where the polar regions have stronger positive anomalies than predicted for a mantle model only (Figure 2h). The splitting function for ${ }_{2} S_{1}$, which has not been observed before, again shows the characteristic 'ring around the Pacific' pattern (Figure 2i), but with a much stronger amplitude than is predicted by S20RTS (Figure 2j).

\subsection{Coefficients}

[13] Our individual $c_{s t}$ coefficients (equation (1)) for $s=2$ are shown in Table 2 and compared with their model predictions and measurements made by previous studies in Figure 3 [He and Tromp, 1996; Resovsky and Ritzwoller, 1998; Masters et al., 2000]. Our new observation for mode ${ }_{0} S_{2}$ (Figure 3a) is almost identical to the model predictions, even though we started our inversion from PREM and thus did not include aspherical mantle structure information. Only $\operatorname{Re}\left(c_{22}\right)$ (index $=4$ ) is different from the model prediction, but this coefficient also has the largest error.
[14] The $c_{20}$ coefficients (index $=1$ ) are generally best resolved. We find that our measured values for $c_{20}$ for modes ${ }_{0} S_{3}$ and ${ }_{0} S_{4}$ (Figures $3 \mathrm{~b}$ and $3 \mathrm{c}$ ) are larger than the predictions for a mantle model but smaller than previous studies. We also find that including cross-coupling for ellipticity and rotation with nearby modes lowers the $c_{20}$ value. Without the cross-coupling, our $c_{20}$ value would have been much more similar to previous studies. The studies by He and Tromp [1996] and Resovsky and Ritzwoller [1998] did not apply cross-coupling for modes ${ }_{0} S_{3}$ and ${ }_{0} S_{4}$, which may explain their larger values. Nonetheless, our values remain larger than the model predictions which may be due to mantle or outer core structure not included in our model.

[15] Our measurements for mode ${ }_{1} S_{2}$ (Figure $3 \mathrm{~d}$ ) are quite different from previous studies, and are much more similar to the model predictions. Since our data set is much larger, we speculate that our measurements are more precise. Again, the most significant difference is for the $c_{20}$ coefficient, which is much larger than the model predictions. ${ }_{1} S_{2}$ is sensitive to the shear wave velocity structure of the inner core. Inner core anisotropy gives rise to anomalous $c_{20}$ values for inner core sensitive modes [Woodhouse et al., 1986], which would explain our observations. Thus, mode ${ }_{1} S_{2}$ should be used with caution when modeling mantle structure.

[16] Splitting function coefficients for mode ${ }_{2} S_{1}$ are much larger than the model predictions (Figure $3 \mathrm{e}$ ). From the damping-versus-misfit trade-off curves (Figure S2), we infer that the larger amplitudes are indeed required by the data. The overall shape of our measurements is still quite similar to the model predictions, indicating that future mantle models may require stronger structure in parts of the mantle.

\subsection{Singlet Frequencies}

[17] Splitting functions for modes ${ }_{0} S_{2}$ and ${ }_{2} S_{1}$ have not been measured before, so we compare our results with previous singlet frequencies measurements for the 2004 Sumatra event [Rosat et al., 2005; Roult et al., 2010]. Singlet frequencies calculated from our splitting functions (Table 3 ) for ${ }_{0} S_{2}$ are very similar to the measurements made by [Rosat et al., 2005] and slightly less similar to those of Roult et al. [2010] (Figure 4a). This similarity is encouraging, especially as we did not include the Sumatra event in our measurement for this specific mode and thus are independent from the previous studies. All measurements have a shape similar to the model predictions, but the frequencies are shifted to higher values than predicted by PREM. This shift agrees with the shift of $0.2 \mu \mathrm{Hz}$ also seen in the centre frequency for ${ }_{0} S_{2}$ in Table 1 .

Table 2. Splitting Function Coefficients (in $\mu \mathrm{Hz}$ ) for $s=2$, With Error Boundaries on the Second Line

\begin{tabular}{cccccc}
\hline Mode & $c_{20}$ & $\operatorname{Re}\left(c_{21}\right)$ & $\operatorname{Im}\left(c_{21}\right)$ & $\operatorname{Re}\left(c_{22}\right)$ & $\operatorname{Im}\left(c_{22}\right)$ \\
\hline${ }_{0} S_{2}$ & -0.66 & -0.09 & 0.02 & 0.06 & -0.25 \\
& 0.32 & 0.47 & 0.18 & 0.87 & 0.38 \\
${ }_{0} S_{3}$ & 0.31 & 0.05 & 0.06 & -0.24 & 0.59 \\
& 0.16 & 0.10 & 0.09 & 0.15 & 0.14 \\
${ }_{0} S_{4}$ & 1.20 & 0.24 & 0.23 & -0.79 & 1.22 \\
& 0.17 & 0.06 & 0.07 & 0.08 & 0.10 \\
${ }_{1} S_{2}$ & 2.02 & -0.17 & -0.05 & -0.69 & 1.22 \\
& 0.15 & 0.28 & 0.17 & 0.38 & 0.38 \\
${ }_{2} S_{1}$ & 2.93 & 1.50 & 1.08 & -5.90 & -1.97 \\
& 0.47 & 1.12 & 0.49 & 0.70 & 1.25 \\
\hline
\end{tabular}




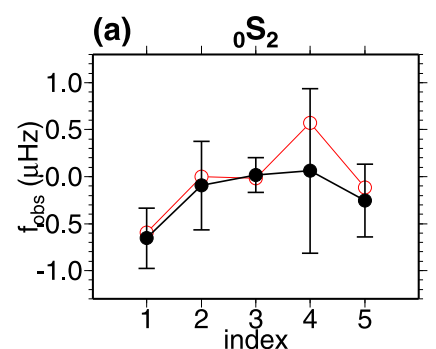

(c)
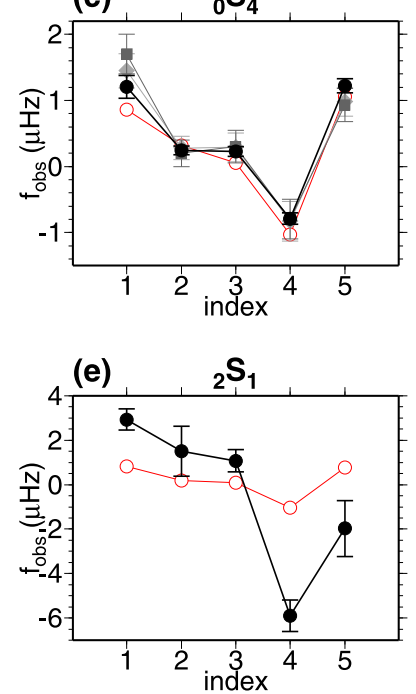

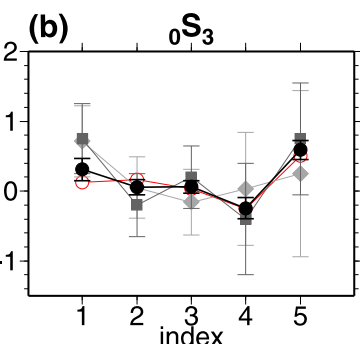

(d)
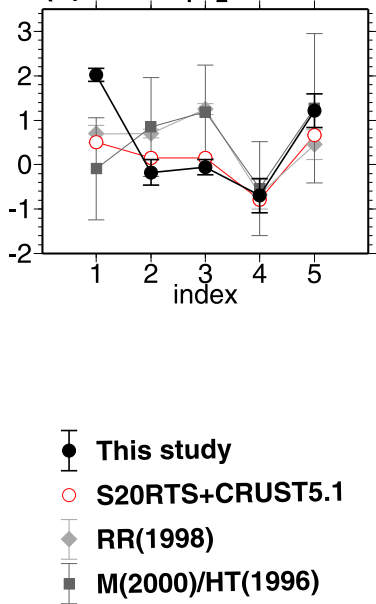
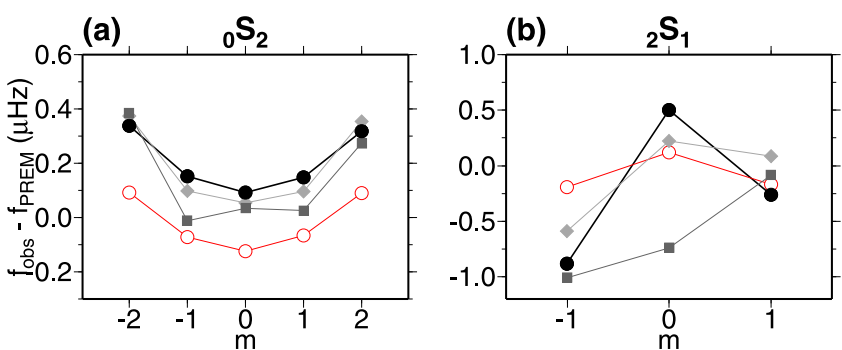

Figure 4. (a, b) Singlet frequencies calculated from our splitting function observations (black circles), compared with S20RTS+CRUST5.1 predictions (red circles) and observations by Rosat et al. [2005] (grey diamonds) and Roult et al. [2010] (grey squares). The frequencies are plotted as deviations from the predictions for PREM using only ellipticity and rotation corrections for the target mode and cross-coupling with its closest neighboring modes.

new mantle shear wave velocity model S40RTS [Ritsema et al., 2010], and will improve constraints on the mantle's heterogeneous density structure.

[20] Acknowledgments. We thank Gabi Laske and Barbara Romanowicz for constructive reviews. AD has been funded by the European Research Council under the European Community's Seventh Framework programme (FP7/2007-2013)/ERC grant agreement 204995. JR is supported by NSF grant EAR-0944167.

Figure 3. (a-e) Observed splitting function coefficients (black solid circles) and the corresponding predictions for mantle model S20RTS and crustal model CRUST5.1 (red circles). Also shown are previous measurements (grey diamonds and squares) by Resovsky and Ritzwoller [1998], Masters et al. [2000] $\left({ }_{0} S_{3}\right.$ and $\left.{ }_{0} S_{4}\right)$ and He and Tromp [1996] ( $S_{2}$ only). The label 'index' denotes the different coefficients, where index $=1$ corresponds to $c_{20}$, index $=2$ is $\operatorname{Re}\left(c_{21}\right)$, index $=3$ is $\operatorname{Im}\left(c_{21}\right)$, etc.

[18] Our singlet frequencies for mode ${ }_{2} S_{1}$ (Figure 4b) are again similar to the measurements by Rosat et al. [2005], but quite different to Roult et al. [2010] for the $m=0$ singlet. All measurements are more strongly split than the model predictions, which was also seen in the splitting function map (Figure 2i) and individual coefficients (Figure 3e).

\section{Conclusions}

[19] Recent earthquakes allow us to improve splitting function measurements of some of the longest period normal modes and make measurements of modes ${ }_{0} S_{2}$ and ${ }_{2} S_{1}$ which had not been possible before. Our results can be easily implemented in tomographic modeling schemes such as our

Table 3. Singlet Frequencies (in $\mu \mathrm{Hz}$ ) for ${ }_{0} S_{2}$ and ${ }_{2} S_{1}$

\begin{tabular}{cccccc}
\hline Mode & $\mathrm{m}=-2$ & $\mathrm{~m}=-1$ & $\mathrm{~m}=0$ & $\mathrm{~m}=1$ & $\mathrm{~m}=2$ \\
\hline${ }_{0} S_{2}$ & 299.93 & 304.63 & 309.28 & 313.86 & 318.40 \\
${ }_{2} S_{1}$ & N/A & 397.92 & 405.18 & 410.45 & N/A \\
\hline
\end{tabular}

\section{References}

Dahlen, F. A., and R. V. Sailor (1979), Rotational and elliptical splitting of the free oscillations of the Earth, Geophys. J. R. Astron. Soc., 58 , 537-549.

Deuss, A., and J. H. Woodhouse (2001), Theoretical free oscillation spectra: The importance of wide band coupling, Geophys. J. Int., 146, 833-842.

Dziewonski, A., and D. Anderson (1981), Preliminary reference Earth model, Phys. Earth Planet. Inter., 25, 297-356.

Edmonds, A. R. (1960), Angular Momentum and Quantum Mechanics, Princeton Univ. Press, Princeton, N. J.

He, X., and J. Tromp (1996), Normal-mode constraints on the structure of the Earth, J. Geophys. Res., 101, 20,053-20,082.

Ishii, M., and J. Tromp (1999), Normal-mode and free-air gravity constraints on lateral variations in velocity and density of the Earth's mantle, Science, 285, 1231-1236.

Li, X.-D., D. Giardini, and J. H. Woodhouse (1991), Large scale three dimensional even degree structure of the Earth from splitting of long period normal modes, J. Geophys. Res., 96, 551-577.

Masters, G., G. Laske, and F. Gilbert (2000), Matrix autoregressive analysis of free-oscillation coupling and splitting, Geophys. J. Int., 143, 478-489.

Mooney, W. D., G. Laske, and G. Masters (1998), A new global crustal model at $5 \times 5$ degrees: CRUST5.1, J. Geophys. Res., 103, 727-747.

Park, J., et al. (2005), Earth's free oscillations excited by the 26 December 2004 Sumatra-Andaman earthquake, Science, 308, 1139-1144.

Resovsky, J. S., and M. H. Ritzwoller (1998), New and refined constraints on three-dimensional Earth structure from normal modes below $3 \mathrm{mHz}$, J. Geophys. Res., 103, 783-810.

Ritsema, J., H. van Heijst, and J. Woodhouse (1999), Complex shear wave velocity structure imaged beneath Africa and Iceland, Science, 286, 1925-1928.

Ritsema, J., H. van Heijst, A. Deuss, and J. Woodhouse (2010), S40RTS: A degree-40 shear velocity model for the mantle from new Rayleigh wave dispersion, teleseismic traveltime, and normal-mode splitting function measurements, Geophys. J. Int., in press.

Romanowicz, B. (2001), How well can we resolve 3D density heterogeneity in the lower mantle?, Geophys. Res. Lett., 28, 1107-1110.

Rosat, S., T. Sato, Y. Imanishi, J. Hinderer, Y. Tamura, H. McQueen, and M. Ohashi (2005), High-resolution analysis of the gravest seismic normal modes after the $2004 \mathrm{Mw}=9$ Sumatra earthquake using superconducting gravimeter data, Geophys. Res. Lett., 32, L13304, doi:10.1029/ 2005GL023128. 
Roult, G., J. Roch, and E. Clevede (2010), Observation of split modes from the 26th December 2004 Sumatra-Andaman mega-event, Phys. Earth Planet. Inter., 179, 45-59.

Tarantola, A., and B. Valette (1982), Generalized nonlinear inverse problems solved using the least squares criterion, Rev. Geophys., 20, 213-232.

Trampert, J., F. Deschamps, J. Resovsky, and D. Yuen (2004), Probabilistic tomography maps chemical heterogeneities throughout the lower mantle, Science, 306, 853-856.

Woodhouse, J. H. (1980), The coupling and attenuation of nearly resonant multiplets in the Earth's free oscillation spectrum, Geophys. J. R. Astron. Soc., 61, 261-283.

Woodhouse, J. H., and D. Giardini (1985), Inversion for the splitting function of isolated low order normal mode multiplets, Eos Trans. $A G U, 66,300$.
Woodhouse, J. H., D. Giardini, and X. D. Li (1986), Evidence for inner core anisotropy from free oscillations, Geophys. Res Lett., 13, 1549-1552.

Zürn, W., G. Laske, R. Widmer-Schnidrig, and F. Gilbert (2000), Observation of Coriolis coupled modes below $1 \mathrm{mHz}$, Geophys. J. Int., 143, 113-1186.

A. Deuss, Bullard Labs, University of Cambridge, Madingley Road, Cambridge CB3 0EZ, UK. (afd28@cam.ac.uk)

J. Ritsema, Department of Geological Sciences, University of Michigan, Ann Arbor, MI 48109, USA.

H. van Heijst, Department of Earth Sciences, University of Oxford, Oxford OX1 3PR, UK. 